\title{
Tipos de riesgos operacionales en empresas metalmecánicas
}

\author{
Types of operational risks in metal-mechanical companies
}

\author{
Patricia Castillo Jiménez \\ patriciacastillojimenez@gmail.com \\ https://orcid.org/0000-0002-6502-7365 \\ Universidad del Zulia, Maracaibo - Venezuela
}

Marle Cecilia Martínez Ramírez

marlemartinez@yahoo.es

https://orcid.org/0000-0002-1531-3320

Universidad del Zulia, Maracaibo - Venezuela

Artículo recibido en abril 2021 / Arbitrado en mayo 2021 / Aceptado en junio 2021 / Publicado en julio 2021

RESUMEN El objetivo de la investigación fue especificar los tipos de riesgos operacionales en las empresas metalmecánicas venezolanas; particularmente aquellas ubicadas en la Costa Oriental del Lago de Maracaibo. La investigación fue de tipo descriptiva, con diseño no experimental, transeccional, de campo. La población estuvo constituida por 11 empresas metalmecánicas, la técnica aplicada fue la encuesta, el instrumento de recolección de datos fue un cuestionario validado por 5 expertos. Se estimó la confiabilidad mediante el coeficiente Alfa Cronbach arrojando un resultado de 0,92 . Se utilizó la estadística descriptiva mediante el análisis de las frecuencias, medias y la desviación estándar. Se concluyó que existe una alta aplicación y presencia de los tipos de riesgos, considerando los indicadores: tecnología de la información, procesos y personas; poniendo en evidencia la expectativa de alta rentabilidad al favorecer que los tomadores de decisiones con reducida aversión al riesgo valoren los beneficios percibidos.

Palabras clave: Gestión de riesgos; Operaciones; Tecnología de la información; Procesos; Riesgo

ABSTRACT The objective of the investigation was to specify the types of operational risks in Venezuelan metalworking companies; particularly those located on the eastern shore of Lake Maracaibo. The research was descriptive, with a non-experimental, cross-sectional, field design. The population consisted of 11 metalworking companies, the technique applied was the survey, the data collection instrument was a questionnaire validated by 5 experts. Reliability was estimated using the Alpha Cronbach coefficient, yielding a result of 0.92. Descriptive statistics was used by analyzing frequencies, means, and standard deviation. It was concluded that there is a high application and high presence of the types of risks, considering the indicators: information technology, processes, and people; highlighting the expectation of high profitability by encouraging decision makers with reduced risk aversion to value the perceived benefits.

Keywords: Risk management; Operations; Information technology; Processes; Risk 


\section{INTRODUCCIÓN}

Toda empresa está expuesta en mayor o menor grado a riesgos que pueden ocasionar daños a personas, medio ambiente, equipos y materiales. La implementación de medidas de mitigación no sólo mejora la capacidad de atención, sino que evita mermas considerables que favorece la operación rutinaria y hace que los sistemas sean más confiables.

En este sentido, las organizaciones afrontan una serie de riesgos que pueden afectar la consecución de sus objetivos. Todas las actividades en una organización están sometidas de forma permanente a una serie de amenazas, que las hace vulnerables, comprometiendo su estabilidad, accidentes operacionales, enfermedades, incendios, pérdidas de beneficios, catástrofes naturales, son una muestra de este panorama, sin olvidar las amenazas propias del negocio.

Visto así, toda empresa se desenvuelve dentro de un ambiente social, económico y político los cuales traen consigo innumerables acontecimientos entre ellos el riesgo operativo, el cual nace como una forma de enfrentar todo el cambio tecnológico de la última generación, creando la conciencia de riesgo en empresas y analizando las principales fuentes de riesgo. Estos traen consigo una serie de repercusiones que tendrán las situaciones críticas y las interrupciones de actividad de las organizaciones podrán variar en gravedad yendo desde pérdidas de poca importancia, como consecuencia de los retrasos de tramitación de pedidos, a la pérdida de reputación o, en el peor de los casos, a la quiebra.

De acuerdo con el Project Management Institute (PMI), el riesgo es un evento o condición incierta que, de producirse, tiene un efecto positivo o negativo en uno o más de los objetivos del proyecto, tales como el alcance, el cronograma, el costo y la calidad (Guía del PMBOK, 2017). Por su parte, la gestión del riesgo en las empresas, más conocida por su término Enterprise Risk
Management (ERM), pretende asegurar que la empresa identifica y gestiona, de la manera más eficaz posible, las diferentes amenazas a las que está expuesta durante el desarrollo de su actividad.

En Venezuela, el sector metalmecánico forma parte importante dentro de la economía nacional, en la región Zuliana particularmente en la Costa Oriental del Lago de Maracaibo; cabe destacar que la industria metalmecánica comprende un diverso conjunto de actividades manufactureras que, en mayor o menor medida, utilizan entre sus insumos principales productos de la siderurgia $y / 0$ sus derivados, aplicándoles a los mismos algún tipo de transformación, ensamble o reparación. Como puede intuirse por su alcance y difusión, la industria metalmecánica constituye un eslabón fundamental en el entramado productivo de una nación; no sólo por su contenido tecnológico y valor agregado, sino también por su articulación con distintos sectores industriales, prácticamente todos los países con un desarrollo industrial avanzado cuentan con sectores metalmecánicos consolidados.

En el contexto actual las empresas metalmecánicas se han visto en la necesidad de ejercer una mejor gestión de riesgos en sus operaciones, tomando en cuenta particularmente el análisis de sus actividades para el mejor desarrollo del proceso de producción que le permita una adecuada planificación, control y ejecución de sus costos.

En virtud de lo expuesto, la investigación busca especificar los tipos de riesgos operacionales, en estas empresas, donde el manejo integral del riesgo y las decisiones de inversión permiten conocer los niveles de incertidumbre a las que están expuestas estas organizaciones durante la ejecución de sus estrategias así como en el cumplimiento de los objetivos planteados, por lo contrario podrían generar problemas en sus operaciones, ocasionando pérdida para la corporación al extremo de su desaparición. 


\section{Gestión de riesgos operacionales}

Con relación a la gestión de riesgos operacionales Fernández (2015) se refiere a ella como un conjunto de metodologías estandarizadas, probadas en muchos mercados que también tienen su aplicación en diferentes niveles de desarrollo de los medios empresariales, a pesar de los escépticos. Sin embargo, la gestión de riesgos no reemplaza nunca el buen sentido común; lo importante es preguntarse qué es un buen sentido común y cómo podría complementarse con la valoración óptima del riesgo.

Asimismo, Bravo y Sánchez (2006) relacionan que la gestión de riesgos operacionales puede ser definida como el proceso mediante el cual se identifican, analizan, evalúan, tratan, monitorean y comunican los riesgos generados en una actividad o proceso, de tal forma que sea posible a las empresas minimizar las pérdidas y maximizar las oportunidades.

De acuerdo con Casares y Martínez (2011), la gestión de riesgos es un campo de trabajo que se encuentra en auge y progresiva profesionalización, el cual puede considerarse como componente transversal para la administración de otros factores relevantes como la calidad, inteligencia estratégica o el cambio organizativo. Hablar sobre gestión de riesgos operacionales ya no se limita al enfoque financiero tradicional o de cobertura. En realidad, esta posee una visión holística de la compañía que contempla aspectos muy variados como la pérdida de control, la seguridad, así como diversas estrategias para prevenir, reducir o transferir el riesgo.

Por su parte Fernández (2015), define el riesgo operacional como un proceso específico dentro de las organizaciones, aporta valor fundamentalmente ya que ayuda a tomar decisiones en relación con el riesgo. Para que dicha toma de decisiones sea eficaz, se deben conocer ciertos aspectos fundamentales de los riesgos, tales como: el impacto potencial en los objetivos, por qué ocurren los riesgos, cuál es su probabilidad de ocurrencia, cuáles son sus consecuencias, con qué medidas cuenta la organización para mitigarlos.

Sin duda todo tipo de empresas, cualesquiera que sean su negocio o su tamaño, deben hacer frente a numerosos retos, tanto internos como externos, los cuales se presenta y pueden conllevar a estas organizaciones no alcanzar los objetivos establecidos. Estos retos e incertidumbres deben gestionarse convenientemente de manera que la actividad de la organización se vea alterada de la menor manera posible por algún evento con consecuencias negativas.

Sobre el asunto Fernández (2015) refiere que la gestión del riesgo en las empresas, conocida por su término sajón Enterprise Risk Management (ERM), pretende asegurar a la empresa identificando y gestionando, de la manera más eficaz posible, las diferentes amenazas expuestas durante el desarrollo de su actividad. Es así como los riesgos operacionales constituyen un nuevo enfoque en las empresas permitiendo gestionar los riesgos para convertirlas en oportunidades, estando mejor preparados para afrontarlos cuando se presente la eventualidad.

El manejo integral del riesgo en empresas, proyectos y decisiones de inversión permite conocer niveles de incertidumbre, durante la ejecución de estrategias y el cumplimiento de objetivos la buena gestión del riesgo es un elemento esencial del buen gobierno empresarial, siendo un instrumento que permite proteger el valor para el accionista. Asimismo, cada vez son más los que reconocen la necesidad de asegurarse que existe un esquema eficaz de controles de la actuación de la dirección.

Endefinitiva,lagestiónderiesgosoperacionales comprende una serie de elementos del sistema de gestión de una empresa, relacionados con la gestión de riesgos. Los elementos pueden incluir una planificación estratégica, toma de decisiones 
y otros procesos que traten el riesgo. De ahí, la cultura de una empresa queda reflejada en su sistema de gestión de riesgos. El beneficio que se puede obtener por cualquier decisión o acción que se adopte, debe asociarse necesariamente con el riesgo inherente a dicha decisión o acción. Los seres humanos deben conocer de una manera intuitiva y cuantitativa a las probabilidades que confrontan en cada decisión. $Y$ hace inferencia en que la esencia de la gestión de riesgo consiste en medir esas probabilidades en contexto de incertidumbre.

Fernández (2015), hace referencia a los riesgos de operaciones como cualquier situación posible que pueda reducir los ingresos de una compañía o que pueda erosionar la imagen ocasionando una pérdida operacional futura, lo que es tratada como riesgo. Por ello es importante aceptar que todas las actividades desarrolladas en una empresa conllevan un riesgo y debe ser correctamente gestionado y medido a fin de garantizar su permanencia en el mercado de manera óptima; la gestión de los riesgos operacionales recae sobre la gerencia de la empresa encargada de controlarlos y mitigarlos.

Con relación a los riesgos operacionales Pardo (2017), la describe como los métodos específicos y puntos a tomar en cuenta en la medición y cuantificación del riesgo operativo así como también las medidas de control específicas para mitigar o disminuir la exposición al riesgo de la empresa o institución.

\section{Tipos de riesgos operacionales}

A continuación, se detallan los tipos de riesgos operacionales a saber: tecnología de la información, procesos y personas orientados por diferentes postulados teóricos.

\section{Tecnología de la información}

La tecnología de la información es el conjunto de tecnologías desarrolladas para gestionar información y enviarla de un lugar a otro, bien puede ser en una empresa (diferentes áreas de productos o servicios), o con otras empresas; con el objetivo de mejorar su competitividad, eficiencia y productividad (De la Peña, 2015). Es así como el uso de estas tecnologías es clave en el mercadeo, ventas, captación de nuevos proveedores, clientes potenciales; permitiendo alcanzar los objetivos organizacionales planeados (Martínez y col., 2017).

Por su parte Fernández (2015) considera la tecnología de la información como un riesgo especial, por cuanto existen varias formas de prevenir esta pérdida de información entre las cuales, la más común y económica es el respaldo de la información cada determinado período de tiempo, si la situación lo amerita inclusive se puede llegar a tener dos sistemas que funcionen en paralelo en lugares físicos distintos con datos actualizados en línea. Cabe destacar que para esto la empresa debe tener un procedimiento por escrito y los empleados responsables están obligados a dominar en detalle dicho procedimiento.

Sobre el asunto Ohia (2018), menciona sobre el avance que la tecnología de la información ha generado hoy en día la plataforma empresarial con base en los sistemas o tecnología de la información, resultando de esto un riesgo muy especial en el contexto de riesgo operativo, puesto que en contraste estos riesgos tienen una naturaleza intangible.

Vinculado al concepto, expresa Morales (2016) que, al tratarse de tecnología de la información, implica la diferencia entre el éxito y fracaso en la vida empresarial, está marcada por la capacidad de la organización de obtener y mantener unos niveles de prestación de números superiores a los de su competencia. Estos niveles se suelen conseguir asociados a mejoras en los sistemas de información que, en muchos casos, se han convertido en una pieza esencial de las operaciones como lo son: software, seguridad de 
la información y de las actividades digitales y los procesos especificados a continuación:

\section{a) Software}

Resultan interesantes las concepciones de Morales (2016) y Ohia (2018), quienes afirman que el software inadecuado o la mala calidad de este puede aumentar considerablemente la exposición al riesgo de una empresa, este punto no es únicamente relativo a un software defectuoso sino también a los problemas y pérdidas que se pueden causar por errores de compatibilidad, configuración, o costos de actualización. El problema más serio que podría suscitarse es el colapso completo del sistema, generando no sólo problemas debido a la pérdida de datos e información sino también debido a retrasos hasta que el sistema esté operativo nuevamente.

Los errores de procesamiento también son peligrosos e inclusive necesitan de un esfuerzo mayor para su determinación y corrección. Inclusive si el software funciona bien se debe analizar el número de usuarios que va a acceder al mismo, el tiempo de cómputo y la calidad de la red.

\section{b) Seguridad de las operaciones}

La seguridad es un factor crucial en los sistemas, existen varios factores que deben ser tomados en cuenta entre los cuales están los accesos no autorizados por terceras personas, que pueden ser con fines de sabotaje, apropiación de datos o información, con fines de obtener ganancias personales, o mucho peor usar la información y modificarla para un beneficio de la tercera persona que ingresa en el sistema (De la Peña, 2015).

El acceso no autorizado de empleados de la empresa o institución, desde su punto básico es un riesgo del personal, pero los sistemas deben presentar las suficientes garantías para evitar o disminuir este tipo de riesgo. Considerando las afirmaciones de Bourgeois (2014), otro punto a tomar en cuenta es el ataque de software maliciosos diseñados para causar daño a los programas, entre estos se encuentran los virus informáticos (se distribuyen pegados a archivos y dispositivos de almacenamiento infectándolos y dañándolos), gusanos (estos tienen la característica de que se propagan mediante la red infectando otros computadores), Trojan Horses (se presentan como programas inofensivos o necesarios para ser instalados por el usuario, pero esconden un propósito real diferente).

Es considerado un riesgo especial de la tecnología de la información a la protección de los datos, por cuanto una insuficiente protección de estos puede generar un riesgo de abuso intencional o falta de cuidado en el manejo. Este riesgo se puede materializar como rectificación o borrado de datos, lo cual puede afectar inclusive a un riesgo reputacional si son datos de clientes.

Las organizaciones se enfrentan al riesgo de quiebras en la seguridad de la información y muy especialmente aquellas que desarrollan una parte importante de su actividad en la red. Del estudio realizado por Espiñeira y col. (2016), aplicado a directivos de importantes compañías, se desprende que prácticamente tres cuartas partes de las empresas participantes habían sufrido este tipo de quiebras en el ejercicio precedente.

Las organizaciones que llevan a cabo actividades de comercio electrónico estaban especialmente en peligro; el $59 \%$ de los participantes que vendían a través de sus sitios Web habían informado, por lo menos, sobre una infracción de la seguridad. Muchos de los participantes no eran capaces de afirmar si habían perdido o no ingresos como consecuencia de las quiebras de seguridad.

Es necesario que las organizaciones tengan la seguridad y certeza el conocimiento de la identidad de sus compradores mediante el comercio electrónico. Necesitan garantías que 
el riesgo de fraude se ha reducido al mínimo y la confidencial en las operaciones. Para ello, pueden recurrir a firmas electrónicas o al uso de nuevos sistemas de identificación únicos como pueden ser el nuevo DNI electrónico.

\section{c) Proveedores externos}

Si bien es cierta la situación de riesgo es compleja cuando el manejo de la información ha sido tercerizado ya sea en parte o el total de ella por una o más compañías, aunque la seguridad recae en otras personas, de alguna manera debe asegurarse del manejo correcto de los sistemas e información (Laudon y Laudon, 2012).

Una falla en las empresas encargadas del manejo de la información no sólo acarrea un riesgo de interrupción del negocio sino también el riesgo de pérdida de datos. Inclusive si no se tiene los derechos de la información y de auditar la misma, se corre el riesgo de perder el control de las actividades fundamentales de la empresa, sin mencionar el know how de la compañía (Bourgeois (2014).

\section{Procesos}

Fernández (2015) define los procesos como la posibilidad de pérdidas financieras relacionadas con el diseño inapropiado de los procesos críticos, o con políticas y procedimientos inadecuados o inexistentes dentro de la empresa, que puedan tener como consecuencia el desarrollo deficiente de las operaciones y servicios o la suspensión de estos.

En tal sentido, podrán considerarse entre otros, los riesgos asociados a las fallas en los modelos utilizados, los errores en las transacciones, la evaluación inadecuada de contratos o de la complejidad de productos, operaciones y servicios, los errores en la información contable, la inadecuada compensación, liquidación o pago, la insuficiencia de recursos para el volumen de operaciones, la inadecuada documentación de transacciones, así como el incumplimiento de plazos y presupuestos planeados.

Por su parte para Buchelt (2006), el estudio del riesgo operacional proveniente de los procesos es el primer paso para distinguir entre los casos que basan su riesgo en la organización del proceso (falla de proceso) y a los que inmiscuyen a las personas encargadas del proceso en sí (error humano) dicho esto se pueden identificar los siguientes riegos en procesos:

\section{a) Cambios de tecnología}

Los cambios de tecnología son decisivos en la incidencia del riesgo operativo en una empresa desde una simple transferencia de información desde un medio a otro más actualizado, hasta la actualización de la máquina principal de la empresa, en este tipo de procesos se pueden dar errores de diferente índole que pueden afectar seriamente a la empresa (Ohia, 2018).

Los cuellos de botella son cruciales en todo proceso ya sea de servicios como de producción, frecuentemente es causado por la falta de recursos, falta de personal especializado o una inadecuada delegación. Además del potencial negativo de los cuellos de botella en la velocidad del proceso, tenemos las consecuencias que podría tener con respecto a toda la línea de producción o de negocio una falla ya sea de índole humana o de los sistemas.

De acuerdo con Morales (2016) los cuellos de botella son cruciales en todo proceso ya sea de servicios como de producción, frecuentemente es causado por la falta de recursos, falta de personal especializado o una inadecuada delegación.

Además del potencial negativo de los cuellos de botella en la velocidad del proceso, se toman en cuenta las consecuencias de una falla ya sea de índole humana o de los sistemas que se podría tener con respecto a toda la línea de producción o del negocio. La redundancia en los procesos trae como consecuencia la reducción de la velocidad 
en el proceso en general, ya que se realizan controles o pasos equivalentes en dos instancias diferentes.

Se entiende por cuello de botella en la gestión de producción como aquel proceso cuya máxima capacidad define la máxima capacidad global. Es decir, una vez que el proceso ha alcanzado su máximo nivel de producción, el sistema no está capacitado para producir más, aunque haya tiempos muertos en el resto del sistema. Este concepto no es exclusivo de la producción, también se aplica en el entorno de la capacidad de servicios y en otros ámbitos.

Una vez identificados los cuellos de botella por uno de los numerosísimos métodos existentes, (el estudio de estos sistemas de identificación escapa de los límites de este proyecto), hay que decidir si se actúa sobre ellos o se mantiene el sistema como está.

Los fallos en los sistemas pueden tener graves consecuencias en los cuellos de botella. Es muy habitual que, en numerosos procesos, sobre todo productivos, se disponga de sistemas en paralelo con el fin de acelerar el nivel de producción. La gestión de los riesgos en este caso debe contemplar la posibilidad que uno de estos dos sistemas en paralelo falle, creando un problema enorme al proceso.

\section{b) Riesgos de proyectos}

De acuerdo con Morales (2016) los proyectos son riesgos específicos que existen sólo por una vez y por un tiempo limitado, pero se debe tener en cuenta los riesgos a los cuales están expuestos; en el peor de los casos el proyecto podría fallar y no sólo desencadenaría en una pérdida de la inversión, también podría afectar a determinada línea del negocio o producción, por lo tanto, se debe tener especial cuidado al analizar este tipo de riesgos.

Expresa por otra parte Pardo (2017) que los proyectos son el vehículo principal empleado por las organizaciones de todo tipo para llevar en la práctica el cambio estratégico y operativo. Son herramientas con múltiples aplicaciones que van desde la creación de un activo material a través de la inversión a la implantación de una amplia variedad de cambios de actividad o la adquisición de una nueva empresa.

Como los proyectos son campos abonados para la creatividad y viveros de oportunidades, y dado que el entorno empresarial se atribuye una elevada importancia al cambio continuo, las organizaciones se ven más impulsadas que nunca a desarrollar una amplia variedad de proyectos en todos los ámbitos; es así como la forma de gestionar un proyecto puede ser la clave de su éxito o fracaso. Uno de los principales problemas de la gestión del riesgo de proyectos es, precisamente, que resulta muy fácil no tomarse suficientemente en serio el proceso de gestión de riesgos. Por ejemplo, es habitual descubrir que aunque se lleva a cabo algún tipo de evaluación de riesgo, ésta se limita a una lista pasiva ubicada en una base de datos que no se emplea activamente, alejándose de la gestión integral recomendada.

\section{Personas}

Los riesgos tratados en este apartado son los relacionados con el personal que trabaja en la empresa, es decir, serán tomados en cuenta sólo los casos en los que errores, omisiones o acciones deliberadas de los empleados generan pérdidas financieras a la empresa. El caso de la influencia de terceras personas es considerado riesgo externo. En este orden de ideas Buchelt (2006), relacionada asocia el riesgo operacional con personas referidos por dos aspectos importantes a saber los principios de ética y el capital humano detallados a continuación:

\section{a) Principios de ética}

La ética profesional encarna los valores y objetivos de una profesión, como la transparencia 
y la rendición de cuentas, la prestación de servicios eficaces de alta calidad y la responsabilidad ante el cliente o consumidor. Es así como el cumplimiento de la ética profesional protege tanto al profesional individual como al honor de la profesión (UNODC, 2019).

El primer riesgo inherente de trabajar con personas es debido a actos criminales de los empleados, normalmente actos cometidos con el afán de obtener ganancias personales o causar daño a la empresa. Entre los casos más importantes se encuentran: el robo o hurto, que consiste en la apropiación de fondos o activos; el fraude, corrupción que va direccionada al acto de aceptar ciertas ventajas por hacer que empleados actúen de determinada manera o por realizar acciones no alineadas a la ética de la empresa o institución y finalmente el conocido como crimen informático que consiste en robo de información, o manipulación de datos de forma deliberada en beneficio propio o a favor o en perjuicio de un tercero ( Fernández, 2015).

\section{b) Capital humano}

Chiavenato (2018) afirma que el talento humano permite las vinculaciones entre las organizaciones exitosas, las cuales reconocen el talento, saben cómo desarrollar las competencias desus integrantes, ylas metas ligadas al crecimiento de negocio, las cuales son remuneradas de acuerdo con el cumplimiento de esos objetivos. Por consiguiente, el talento humano proporciona la energía creativa de la organización.

Todas las actividades humanas están expuestas a errores y mientras más compleja es la actividad, más alto es el riesgo. El panorama de este tipo de riesgos es muy amplio incluyen ingresos de datos o procesos incorrectos por falta de experiencia o conocimiento. Cabe destacar que en contraste con los actos criminales estos errores $\mathrm{u}$ omisiones son causados por negligencia, o falta de experiencia ya que de otra manera serían considerados actos dolosos.
Los riesgos del personal se ven incrementados cuando no hay el suficiente capital humano para determinado trabajo, dando como resultado una excesiva carga de trabajo para los empleados existentes, incrementando los cuellos de botella y la presión en los trabajadores lo cual guía a un aumento de estrés y frustración en los mismos. La falta de personal en determinada área del negocio puede ser debido ya sea a un gran crecimiento del área o a medidas no técnicas de reducción de personal (Chiavenato, 2018).

Si el planeamiento de personal no toma en cuenta la posibilidad de una enfermedad o renuncia de alguno de los empleados, los problemas por falta de colaboradores se podrían ver seriamente incrementados ya que con la persona que deja la institución se va todo el know how y las habilidades adquiridas en su trabajo a lo largo del tiempo.

Existen casos donde un empleado se convierte en una fuente potencial de riesgo debido a su posición y habilidades, esto no hace referencia a la falta de experiencia para cumplir su trabajo, pero por su estatus de extraordinario tiene la facilidad de saltar barreras y controles con la autorización inclusive de sus superiores para realizar su trabajo ya que éste genera excelentes resultados a la empresa, por ello se debe determinar el riesgo de sus acciones e inversiones por cuanto a mayor rentabilidad siempre se corren mayores riesgos y también la empresa está más expuesta al fraude (Morales, 2016).

Por su parte Fernández (2015), precisa el riesgo operacional de personas como la posibilidad de pérdidas financieras asociadas con negligencia, error humano, sabotaje, fraude, robo, paralizaciones, apropiación de información sensible, lavado de dinero, inapropiadas relaciones interpersonales y ambiente laboral desfavorable, falta de especificaciones claras en los términos de contratación del personal, entre otros factores. Se puede también incluir pérdidas asociadas con 
insuficiencia de personal o personal con destrezas inadecuadas, entrenamiento y capacitación inadecuada y/o prácticas débiles de contratación.

Otro criterio por considerar es el expresado por Morales (2016) los cuales, visualizan este tipo de riesgo están muy relacionados con el desarrollo personal, cuidar de ellos es una necesidad raramente cumplida. Hoy día muchas empresas dependen de ciertas personas que son las únicas con autoridad o conocimientos para realizar determinadas actividades, debido a ello se deben intentar estrategias a fin de alcanzar los máximos niveles de motivación y prevenir el riesgo de que estas personas no rindan como se espera, se desmotiven o renuncien de la empresa.

Es conveniente que no sea un solo empleado especializado en una determinada función y, si es así, instruir a otro o ante la máxima que nadie debe ser imprescindible en la empresa. Para paliar el ausentismo laboral, las organizaciones han diseñado sistemas y métodos para aumentar la motivación de sus empleados logrando así que la productividad por empleado aumente.

Asimismo, Morales (2016) afirma que para desarrollar un completo proyecto empresarial hay que valorar tanto o más el aspecto humano como el técnico y el logístico. Es indudable que gestionar el riesgo con términos numéricos y palpables o que sean fácilmente auditables supone una simplificación enorme del trabajo, sin embargo, los errores en las relaciones entre empleados o en la motivación, entre otros, son los que antes se detectan.

\section{MÉTODO}

La investigación se enmarca en el tipo descriptivo, corresponde a un diseño no experimental, transeccional y de campo (Arias, 2016). La población de estudio estuvo conformada por 11 empresas del sector metalmecánico de la Costa Oriental del Lago de Maracaibo, representadas por 22 sujetos informantes (gerentes y supervisores) a quienes se les aplicó el instrumento de recolección de datos. La validez se calculó a través del juicio de cinco (5) expertos, con relación a la confiabilidad se obtuvo un 0,92 mediante el Coeficiente Alfa Cronbach indicando muy alta confiabilidad del instrumento. Por su parte, para el tratamiento de los resultados se recurrió al método de la estadística descriptiva específicamente el análisis de la media aritmética, la distribución de frecuencias y la desviación estándar.

Para el análisis de las frecuencias se consideró conveniente fijar un baremo de interpretación, el cual se expresa en la Tabla 1, se muestra el rango en donde pueden darse los resultados con base a la suma de las respuestas más positivas siempre y casi siempre $(\mathrm{S}+\mathrm{CS})$, se consideraron de APLICACIÓN y los ítems cuyas respuestas fueron Algunas Veces, Casi Nunca y Nunca, se consideraron de NO APLICACIÓN; de la sumatoria indicada se obtienen las frecuencias absolutas y relativas de cada ítem; de allí se calculó el porcentaje de aplicación para cada indicador, su categoría asignada e interpretación. Seguidamente se presenta la Tabla 2 , perteneciente al baremo para la interpretación de la media aritmética y la Tabla 3 , referido al análisis para la interpretación de la desviación estándar. 
Tabla 1. Interpretación del porcentaje de la distribución de frecuencias.

\begin{tabular}{ccc}
\hline RANGO & CATEGORÍA & INTERPRETACIÓN \\
\hline$\geq 80,01$ a $100 \%$ & Muy alta aplicación & Implica muy alta fortaleza para las empresas. \\
$\geq 60,01$ a $80 \%$ & Alta aplicación & Implica alta fortaleza para las empresas. \\
$\geq 40,01$ a $60 \%$ & Moderada aplicación & Implica moderada fortaleza para las empresas. \\
$\geq 20,01$ a $40 \%$ & Baja aplicación & Implica alta debilidad para las empresas. \\
0 a $20 \%$ & Muy baja aplicación & Implica muy alta debilidad para las empresas. \\
\hline
\end{tabular}

Tabla 2. Baremo para la interpretación de la media aritmética.

\begin{tabular}{ccc}
\hline INTERVALO & CATEGORÍA & INTERPRETACIÓN \\
\hline $4,21-5,00$ & Muy alta presencia & Indica muy alta presencia del indicador. \\
$3,41-4,20$ & Alta presencia & Indica alta presencia del indicador. \\
$2,61-3,40$ & Moderada presencia & Indica moderada presencia del indicador. \\
$1,81-2,60$ & Baja presencia & Indica baja presencia del indicador. \\
$1,00-1,80$ & Muy baja presencia & Indica muy baja presencia del indicador \\
\hline
\end{tabular}

Tabla 3. Análisis para la interpretación de la desviación estándar.

\begin{tabular}{|c|c|c|}
\hline INTERVALO & CATEGORÍA & INTERPRETACIÓN \\
\hline $1,61-2,00$ & Muy alta & $\begin{array}{l}\text { Indica una muy alta dispersión de las respuestas y, una } \\
\text { muy baja confiabilidad de estas. }\end{array}$ \\
\hline $1,21-1,60$ & Alta & $\begin{array}{l}\text { Indica una alta dispersión de las respuestas y, una baja } \\
\text { confiabilidad de estas. }\end{array}$ \\
\hline $0,81-1,20$ & Moderada & $\begin{array}{l}\text { Indica una moderada dispersión de las respuestas y, } \\
\text { una moderada confiabilidad de estas. }\end{array}$ \\
\hline $0.41-0,80$ & Baja & $\begin{array}{l}\text { Indica una baja dispersión de las respuestas y, una alta } \\
\text { confiabilidad de estas. }\end{array}$ \\
\hline $0-0,40$ & Muy baja & $\begin{array}{l}\text { Indica una muy baja dispersión de las respuestas y, } \\
\text { una muy alta confiabilidad de estas }\end{array}$ \\
\hline
\end{tabular}

RESULTADOS

A continuación, se presentan en la tabla 1 los resultados obtenidos correspondientes a tipos de riesgos operacionales en empresas metalmecánicas de la Costa Oriental del lago de Maracaibo, representada por cuatro dimensiones, con sus respectivos indicadores con un promedio de $68 \%$ con alta fortaleza.
Deacuerdoconlos resultadoscorrespondientes a la dimensión tecnología de la información, el indicador software refleja un promedio de $77,3 \%$ representado un nivel de alta aplicación/alta fortaleza, una media de 4,29 muy alta presencia; una dispersión de 0,39 muy alta confiabilidad. En lo que respecta al indicador seguridad de las operaciones se expresa un promedio de $54,5 \%$ 
con un nivel de moderada aplicación/moderada fortaleza; una media de 4,25 muy alta presencia, con una dispersión de 0,42 alta confiabilidad. Por su parte el indicador proveedores externos se muestra un $36,4 \%$ con nivel de baja aplicación/ alta debilidad; media de 3,02 moderada presencia y una dispersión de 1,06 moderada confiabilidad.

Ahora resumiendo los indicadores se observó el $56 \%$ representado como una moderada aplicación/moderada fortaleza, la media alcanzó un 3,85 demostrando alta presencia con una dispersión de 0,62 alta confiabilidad; los resultados revelan que los softwares utilizados en la mayoría de las empresas metalmecánicas son analizados antes de su implementación, asimismo se adaptan a las necesidades de estas.

Tabla 4. Tipos de riesgos operacionales en empresas metalmecánicas de la Costa Oriental del Lago de Maracaibo.

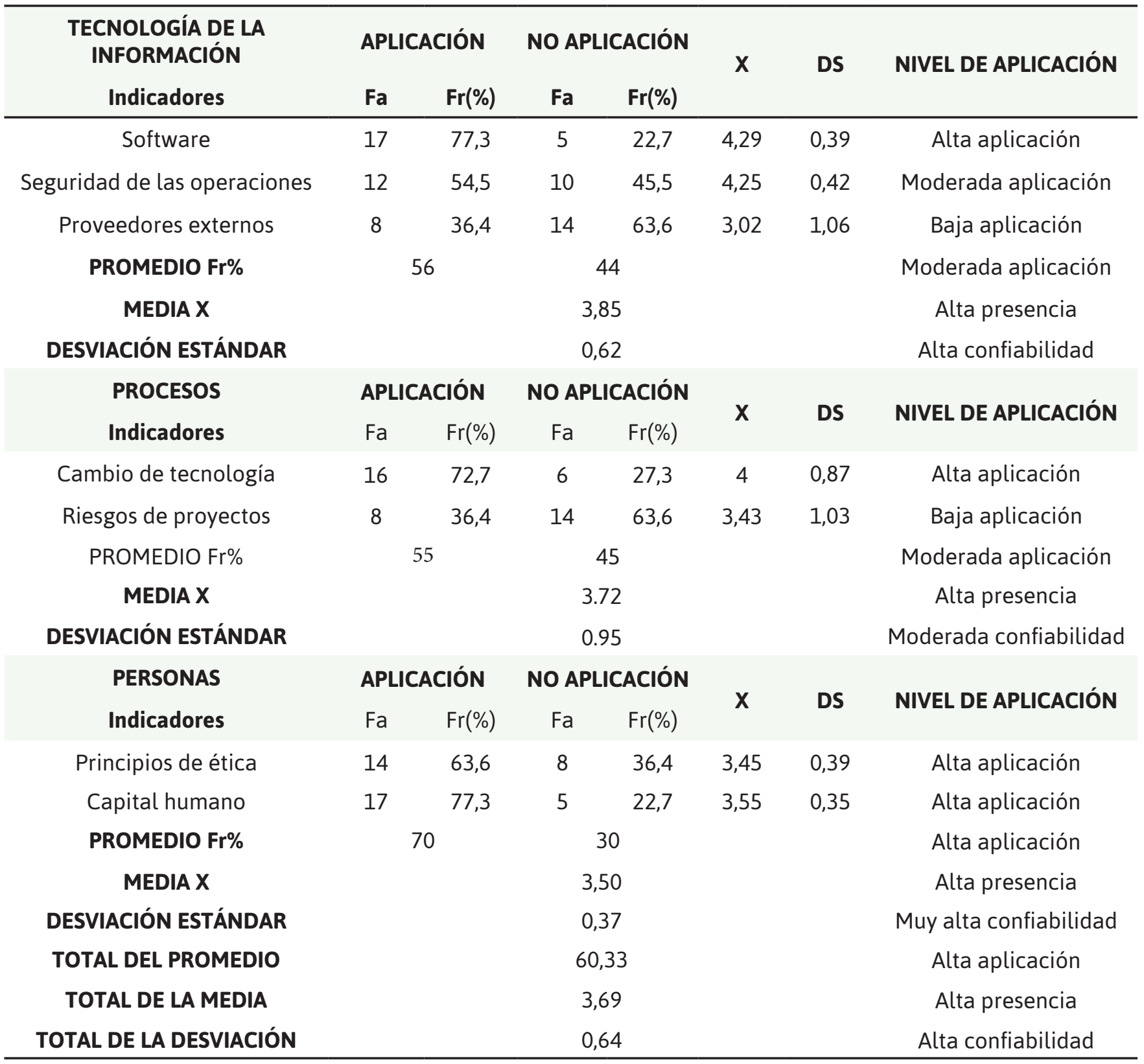


Cabe destacar que de acuerdo con De la Peña (2015), Ohia (2018) es clave la plataforma tecnológica de una empresa, y sus sistemas o tecnologías de la información, resultando de esto un riesgo muy especial dentro del contexto de riesgo operativo, puesto que en contraste tienen una naturaleza intangible, sin embargo, los aspectos principales a tomar en cuenta según la autora son: software inadecuado, seguridad, interrupción de operaciones día a día y proveedores externos.

Con respecto a los proveedores externos en las empresas metalmecánicas de la Costa Oriental del lago de Maracaibo se evidencia que deben preocuparse por manejar proveedores externos confiables, ajustándose a las necesidades y requerimientos del área operacional por observar un resultado de baja aplicación/baja fortaleza. Por lo tanto, tienen un bajo nivel de validación con relación a los postulados de Laudon y Laudon (2012) y Bourgeois (2014), las cuales limitan sus accesos a personal autorizado por área según lo afirmado por Morales (2016) relaciona, la tecnología de la información, como la diferencia entre el éxito y el fracaso en la vida empresarial actual, está marcada por la capacidad de la organización de obtener y mantener unos niveles de prestación de números superiores a su competencia.

Continuando con el análisis se presentan los resultados de la dimensión procesos; el indicador cambio de tecnología muestra un $72,7 \%$ nivel de alta aplicación/alta fortaleza; una media de 4 alta presencia, y una dispersión de 0,87 moderada confiabilidad. Por su parte el indicador riesgos de proyectos alcanzó un $36,4 \%$ baja aplicación/alta debilidad; una media de 3,43 alta presencia, con una dispersión de 1,03 baja confiabilidad.

Ahora bien, resumiendo los indicadores cambio de tecnología y los riesgos de proyectos se obtuvo un nivel de aplicación del 55\% expresando moderada aplicación/moderada debilidad; la media con un valor de 3,72 alta presencia y finalmente una dispersión de 0,95 moderada confiabilidad. De acuerdo con los procesos de una empresa son estudiados como la posibilidad de pérdidas financieras relacionadas con el diseño inapropiado de los procesos críticos, o con políticas y procedimientos inadecuados 0 inexistentes dentro de la empresa, que puedan tener como consecuencia el desarrollo deficiente de las operaciones y servicios o la suspensión de estos.

Los resultados obtenidos validan lo expresado por Morales (2016) y Ohia (2018), Fernández (2015), para quienes el cambio de tecnología son decisivos en la incidencia del riesgo operativo, por cuanto los fallos en los sistemas pueden tener graves consecuencias; con relación al riesgo del proyecto, los resultados demuestran bajo nivel de validación considerando los fundamentos de Morales (2016) y Pardo (2017) para quienes los proyectos son riesgos específicos que existen sólo por una vez y por un tiempo limitado, y representan el vehículo principal para llevar en la práctica el cambio estratégico y operativo por las organizaciones a fin de asumir un riesgo particular.

En función con los resultados de la dimensión personas el indicador principios de ética representado por un $63,3 \%$ mostró un nivel de alta aplicación/alta fortaleza, la media de 3,45 alta presencia y una dispersión de 0,39 muy alta confiabilidad. Por su parte el indicador capital humano arrojó un $77,3 \%$ expresando una alta aplicación/alta fortaleza, la media de 3,55 alta presencia con una dispersión de 0,35 alcanzando una muy alta confiabilidad.

En vista de lo anterior y resumiendo la dimensión se obtuvo un promedio de $70 \%$ representando una alta aplicación/alta fortaleza, una media de 3,50 alta presencia y una dispersión de 0,37 alta confiabilidad. Las ideas anteriormente expuestas validan con los autores Chiavenato (2018), Fernández (2015) y Morales (2016) se puede inferir que este tipo de riesgo operacional representa una vital importancia para las 
empresas al contar con un personal con un alto nivel ético e identificado con la cultura de la organización garantizando un alto rendimiento a nivel productivo y desenvolvimiento en su área de trabajo.

Finalmente resumiendo los resultados referidos a los tipos de riesgos operacionales en empresas metalmecánicas de la Costa Oriental del Lago de Maracaibo se arriba a un total del promedio de 60,33 indicando alta aplicación representando una alta fortaleza; con un total de la media 3,69 alta presencia y una dispersión de 0,64 alta confiabilidad.

Estas evidencias coinciden y validan las teorías expuestas por Buchelt (2006), Morales (2016), Fernández (2015) y Pardo (2017), Chiavenato (2018); donde los resultados evidencian que las empresas metalmecánicas de la Costa Oriental del Lago de Maracaibo objeto de estudio se atribuyen alta importancia al reconocer los tipos de riesgos presentes en sus operaciones a partir de la tecnología de la información, los procesos y las personas; en cuanto a las consecuencias, la preferencia de los gestores por comportamientos arriesgados está asociada positivamente con el logro de elevados resultados.

\section{CONCLUSIONES}

Una adecuada gestión de los riesgos organizacionales aumenta la probabilidad de alcanzar objetivos de la empresa. Tiene una importancia estratégica como herramienta para el logro de mejores resultados en el desempeño de la organización sobre la base de la información objetiva con datos controlados, la cual disminuye la incertidumbre de los procesos para cumplir con su objeto social, tomando las medidas preventivas para disminuir tanto la frecuencia de ocurrencia de eventos indeseables como sus impactos.

El manejo integral del riesgo en las empresas, proyectos y decisiones de inversión permite conocer niveles de incertidumbre a la que están expuestas las organizaciones, durante la ejecución de estrategias y el cumplimiento de objetivos la buena gestión del riesgo es un elemento esencial del buen gobierno empresarial, siendo un instrumento que permite proteger el valor para el accionista.

En efecto el riesgo ha sido tradicionalmente tratado en todas las organizaciones, aunque se realizara de manera indirecta. En líneas generales para todo tipo de actividad económica existe el denominado riesgo operacional, el cual se deriva de las decisiones que en el seno de la empresa se toman diariamente, ya sea en relación con la producción, distribución, precios, entre otros.

Una vez finalizada la investigación y en función de los resultados obtenidos se especificaron los tipos de riesgos operacionales en las empresas metalmecánicas, se concluye que los tipos de riesgos poseen alta aplicación, con promedio de frecuencias en las opciones positivas que las categorizaron como una alta fortaleza.

Sin embargo, al detallar los indicadores de la dimensión tecnología de la información se evidenció una alta debilidad con relación a los proveedores externos; con relación a la dimensión procesos el indicador correspondiente a los riesgos de proyecto arrojó una alta debilidad. Por su parte la dimensión personas en sus indicadores principios de ética y capital humano alcanzaron una alta fortaleza.

Es oportuno aclarar que esta situación de alta presencia es el resultado que las empresas metalmecánicas bajo estudio están aplicando los tipos de riesgos operaciones durante la ejecución de sus procesos, mostrando alta fortaleza a nivel de software, cambio de tecnología y el nivel de su personal. Se resalta entonces, que algunos tipos de riesgos fueron visualizados con moderada y alta fortaleza, no alcanzando el nivel de muy alta presencia/muy alta fortaleza, indicando que estas empresas pueden ofrecer oportunidades de mejora. 
Finalmente, los gestores de proyecto suelen estar muy ocupados, atendiendo a patrocinadores, proveedores, elaborando informes de avances, y frecuentemente, es necesario dar una nueva definición a la función del gestor de riesgo, para que éste no se limite a evitar el riesgo en sí mismo, sino a conseguir que los procesos funcionen mejor. Es decir, la gestión del riesgo tiene que ser más proactiva y menos reactiva en la mayoría de los proyectos. Por tanto, es necesario atribuir la responsabilidad sobre los riesgos, y los planes de acción a seguir a una persona que debe adoptar conscientemente la decisión de asumir un riesgo particular.

\section{REFERENCIAS}

Arias, F. (2016). El proyecto de investigación: Introducción a la metodología científica. Caracas: Editorial Episteme

Bourgeois, D. (2014). Information Systems for business and beyond. Estados Unidos: Editorial Biola University

Bravo, I y Sánchez, M. (2006). Gestión integral del riesgo. Bogotá: Editorial consorcio gráfico Ltd.

Buchelt, R. (2006). Control y provisión de sistemas y procesos de las personas. México: Editorial Otto Wagner Platz 3

Casares, I y Martínez, M. (2011). El proceso de gestión de riesgos como componente integral de la gestión empresarial. Bilbao: Boletín de Estudios Económico

Chiavenato, I. (2018). Administración de recursos humanos. México: Mc Graw Hill Interamericana

De la Peña, Natalia. (2015). Gestión y control de los sistemas de información. España: Editorial Elearning

Espiñeira, Sheldon y Asociados. (2016). Boletín de Asesoría Gerencial. Gestión de Riesgo Operacional. Caracas Venezuela

Fernández, A. (2015). La gestión del riesgo operacional: Aplicación. Editorial Universidad de Cantabria. Madrid. España
Laudon, Kenneth., y Laudon, Jane. (2012). Sistemas de Información Gerencial. México: Pearson Educación

Morales, R. (2016). Fundamentos de BPMN: Una Guía Básica para el Diseño de Procesos. España: CreateSpace Independent Publishing Platform

Martinez, Marle., Serra, Luisa., Brenzini, Daniela., Mármol, María., y Chirinos, Jelvis (2017). Elementos de las tic en empresas de servicio petrolero. Caso venezolano. Impacto Científico. Revista Arbitrada Venezolana del Núcleo LUZCosta Oriental del Lago. Vol. 12. N¹. Junio 2017. pp.13-23

Ohia, N. (2018). Biblioteca de riesgo y vulnerabilidad de infraestructura de TI: Un registro consolidado de vulnerabilidades de infraestructura operativa y tecnológica. España: Independently Published

Pardo, J. (2017). Gestión por procesos y riesgo operacional. Asociación Española de Normalización y Certificación. Madrid: AENOR Ediciones

Project Management Institute (2017). La guía de los fundamentos para la dirección de proyectos (Guía del PMBOK). Pennsylvania: Inc, editor

UNODC (2019). OFICINA DE LAS NACIONES UNIDAS CONTRA LA DROGA Y EL DELITO EDUCACIÓN PARA LA JUSTICIA. Serie de módulos universitarios. Integridad y Ética. Viena. Austria. Recuperado el 20 de abril de 2020 de: https://www.unodc.org/documents/e4j/ IntegrityEthics/MODULE_14_-_Professional_ Ethics_-_Spanish_v.pdf 